\title{
CONTEXTUAL REPRESENTATION MAY MEDIATE SEX DIFFERENCES IN HETEROSEXUAL ATTRACTION
}

\author{
ANDREW WATt " , DEINIOL SKILliCORN, JEDIAH CLARK, RACHEL EVANS, \\ Paul HeWlett ANd NiCK Perham
}

\author{
Cardiff Metropolitan University
}

Received: 2 July 2014; accepted: 20 June 2015

\begin{abstract}
Background: Heterosexual men and women differ in their sensitivity to cues indicating material status. This dissociation has been explained by appealing to sexual selection processes that encourage women to evaluate men on the basis of their material status but could perhaps be explained by sex differences in contextual attention, or, associative representations.

Method: In Experiment 1, heterosexual women rated the attractiveness of an opposite sex model in 4 conditions; (1) attractive context, (2) attractive context with implied ownership, (3) unattractive context, and (4) unattractive context with ownership implied. A second experiment used a fictitious stockbroker learning task (with both men and women) in 2 biconditional discriminations to measure contextual attention (stage 1) and then to explore the structure of contextual representation (stage 2) using a transfer of occasion setting test.

Results: In Experiment 1, females increased ratings in attractive contexts, both when context ownership was implied and when it was not. In the first stage of Experiment 2, men and women were equally sensitive to contextual cues. In stage 2, women's learning was impaired when a stimulus previously used as a target was employed as a context (they showed transfer of occasions setting), men showed no such difference.

Conclusions: Sex differences in sensitivity to cues indicating material status may reflect how men and women tend to encode the relationships between background/context stimuli and target stimuli. Women automatically attend to the background and modulate the value of targets using a hierarchical form of representation, whilst men represent background-target associations configurally.
\end{abstract}

Keywords: biconditional discrimination, ocassion setting, hierarchical representation, configural representation, background attractiveness

\section{INTRODUCTION}

It has been argued that men evaluate attractiveness on the basis of the physical characteristics of women (e.g., hip to waist ratio, Furnham et al., 2006) whilst women base their evaluations on cues that indicate wealth and status (Aharon et

* Corresponding author: Dr Andrew Watt; Department of Applied Psychology, Cardiff Metropolitan University, Western Avenue, Cardiff, Wales, CF5 2YB, UK; E-mail: awatt@cardiffmet.ac.uk

This is an open-access article distributed under the terms of the Creative Commons Attribution License, which permits unrestricted use, distribution, and reproduction in any medium for non-commercial purposes, provided the original author and source are credited. 
al., 2001; Buss, 1989). Research examining human attraction has been dominated by evolutionary interpretation, with human mating preferences explained primarily in terms of sexual selection of status sensitivity in women and identification of reproductive fitness by men (Buss, 1989; Buss \& Schmitt, 1993; Townsend, 1989). Sex differences in mate selection criteria are thought to arise because of the asymmetrical costs of reproduction for men and women. Women tend to evaluate men on the basis of their control of resources necessary for successful long-term nurturing (e.g., Trivers, 1972).

Some researchers have directly manipulated the perceived material status of models through changes of costume (Hill, Nocks, \& Gardner, 1987; Hoult, 1954) or apparent ownership of prestige cars/apartments (Dunn \& Searle, 2010; Dunn \& Hill, 2014). Both of these manipulations have shown that women are sensitive to status manipulations (attractiveness of a model increases in the high status condition), whilst men are not. These findings are consistent with the argument that women base mate selection choices on material status more than on physical attractiveness (Trivers, 1972). Sexual selection accounts must suppose that evolutionary pressures have resulted in women developing traits that increase sensitivity to contextual cues that convey information about material status and must also modify their appraisals of attractiveness on the basis of this information.

It follows from sexual selection based accounts that, because female attractiveness ratings are specifically influenced by material status, then such effects should be confined to conditions where ownership of valued artefacts or resources can be reasonably inferred by the woman performing the evaluation. Female ratings of attractiveness, however, have been shown to be sensitive to a range of non-specific factors including morals, personality, hobbies and education (Gibbins, 1969). One way to provide a common conceptualisation of these factors (including high status ownership) is that they may all act as background information (or contextual stimuli) that women tend to include during their assessments of opposite sex attractiveness. The cognitive psychology literature provides good reasons to suppose that men and women differ in terms of their sensitivity to contextual, background or peripheral information.

Perhaps because of the evolutionary focus in the literature, explanations of the sex differences in status sensitivity have not hitherto considered the operation of more general cognitive or attentional processes that have been shown elsewhere to differ between men and women. In fact, sex differences have been reported in a range of cognitive abilities. In the field of selective attention, differences between men and women have been reported in a variety of domains (Halpern, 2000). Women have been shown to direct more attention to invalid distractor cues than men in vigilance tasks (Merritt et al., 2007). Men show superior performance on attentionally focused visuo-spatial tasks (Collins \& Ki- 
mura, 1997) and men also have an advantage in tests of sustained attention (Giambra \& Quilter, 1988).

In contrast, women perform better in tests of episodic memory (Yonker et al., 2003). Women therefore seem to have an advantage in tasks that require wide attentional capture, whilst men perform better in situations requiring narrow attentional focus.

Further evidence for sex differences in contextual attention comes from the eyewitness testimony literature where women often show superior focus on peripheral detail, providing more elaborate recall for non-central information such as decor, hair colour, hair length, jewellery, and weight (Yarmey, 1986, 2004). Perhaps most relevant to the current study is the observation, in cueing paradigms, that attention in women is directed more by the background than it is in men (Bayliss et al., 2005). One interpretation of the above differences is that men ignore irrelevant background cues more efficiently, whilst women tend to encode background stimuli automatically. It follows that men may potentially have a decrement in attention for context, perhaps even when the context is relevant. Under such circumstances it is reasonable to suppose that women would have an advantage. In eyewitness testimony for example, peripheral or background details may be important when corroborating perpetrator identification or the relative timing of events.

It is clear that sex differences reported in the cognitive psychology literature are consistent with those in evolutionary psychology, however the 2 disciplines disagree about the likely sources of these differences. It has been reported, for instance, that men direct attention to a potential mate's physical appearance (Hassebrauk, 1998) which, it could (from a cognitive psychology perspective) be argued, reflects relatively focused attention. In contrast, womens' evaluations of attractiveness seem to be mediated by context cues (e.g., Dunn \& Searle, 2010; Dunn \& Hill, 2014), suggesting (from a cognitive psychology perspective) the engagement of more diffuse processes and wider attentional capture. It is plausible therefore (from a cognitive psychology perspective) that apparent status-based sex differences in attraction could reflect one of many instances of sex differences in automatic attentional capture.

The above cognitive-contextual account and status-sensitivity accounts make quite different predictions regarding how women's attractiveness ratings will be influenced by simultaneous manipulations of background attractiveness and implied ownership. The cognitive psychology account supposes that women should rate males as more attractive in any attractive context, because they automatically attend to, and are influenced by, background stimuli. In contrast, the material status account supposes that women are specifically influenced by implicit ownership of objects, ratings should increase only when an object implying increased material status (e.g., large expensive-looking house) is present, 
ratings should not increase in attractive contexts where there are no high status objects (e.g., in a beautiful landscape). It follows that sexual selection accounts predict a 2-way interaction between background attractiveness and implied ownership in women because differences for women are only anticipated between conditions where there are artefacts that could potentially be owned. The purpose of the first experiment was therefore to manipulate both background attractiveness and implied ownership in order to determine the status-specificity of the mediation of attractiveness assessments in heterosexual women.

\section{METHOD FOR EXPERIMENT 1}

\section{Design}

A repeated measures design was used with the independent variables of context (attractive / unattractive) and implied ownership (own object / own nothing). The dependent measure was the attractiveness ratings of the target model who the participants rated on a scale of 1 (highly unattractive) to 10 (highly attractive).

\section{Selection of materials}

In order to ensure intermediate attractiveness for the target male, six participants ( 3 female and 3 male) were presented with a pool of 13 male potential target models. All of the target models at this stage were presented against a uniform white background. The participants were asked to rate the potential targets in terms of sexual attractiveness. The model rated closest to the midpoint of 5 on the rating scale (1-10) was chosen to be the target model in the first experiment.

In order to ensure that the manipulated contexts fitted the labels 'attractive' and 'unattractive', all photographs of contexts were assessed for their attractiveness without super-imposition of the model photographs using the same raters.

\section{Participants and ethics}

Sixty four female students were initially invited to participate in Experiment 1. Ratings from 4 women were excluded from analysis because they reported a gay sexual orientation. Sixty women were included in the study sample, their ages ranged between 18 and 25 years and they were recruited from university lecture theatres. All participants gave informed consent and were free to with- 
draw from the study at any time. The procedure was granted approval by the Cardiff School of Health Sciences ethics panel.

\section{Procedure}

Data were obtained over a 1 week period. Once the women had consented to participate, they were then asked to sit separately in a teaching room at the university. Participants were shown a slide show using Microsoft PowerPoint that contained a total of 64 photographs. Participants were asked to report their sexual orientation and heterosexual orientation was an inclusion criterion.
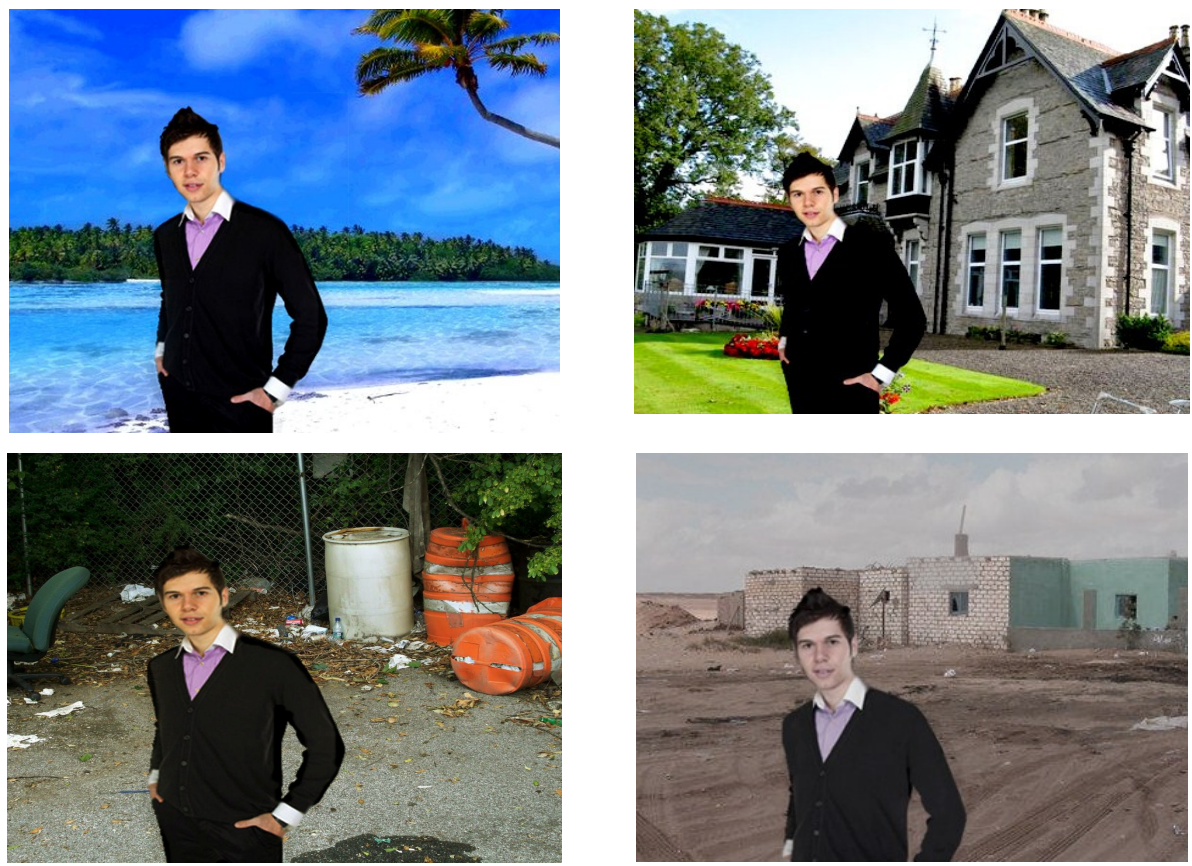

Figure 1. Images of the male model and backgrounds presented to participants in Experiment 1. Participants were presented with each photograph featuring the model in attractive contexts with (top right) and without (top left) implied ownership and in unattractive contexts with (bottom right) and without (bottom left) implied ownership

The procedure for stimulus presentation was consistent with that previously reported by Tractinsky et al. (2004). Specifically, each slide was presented for 1 second and participants were then given 5 seconds to rate the target model 
for attractiveness on a scale of 1-10 using a response booklet. Target models were presented super-imposed against four context backgrounds (attractive context (e.g., beautiful landscape), unattractive context (e.g., waste dump), attractive context implying ownership (e.g., large expensive-looking house) and unattractive context implying ownership (e.g., run down house) (see Figure 1). Twenty eight male distracter photographs were included in order to conceal the purpose of the study, each of these was presented twice in order to minimise the possibility of participants detecting that the target stimuli were different from distractors by virtue of multiple presentation. Each of the models was presented twice in each of the manipulated contexts. The participants therefore received a total of 8 manipulated context slides ( 2 of each) and 56 distractor slides. The order in which the target models appeared in the PowerPoint presentation was arranged so that at least four distracter photos separated consecutive presentations of the target stimuli.

Participants were instructed to rate the target model for attractiveness and no emphasis on the context was given. Ratings for the distracter photographs were discarded from analysis.

\section{RESULTS}

Mean attractiveness ratings for the four conditions of the experiment are presented in Figure 2. The attractiveness ratings of the women were not uniform across the 4 conditions of Experiment 1, attractiveness ratings appeared higher when the model had been superimposed on attractive backgrounds, both when ownership was implied (consistent with sexual selection hypotheses) and when it was not (inconsistent with sexual selection accounts).

The attractiveness ratings across the 4 conditions of Experiment 1 were subjected to a 2-way ANOVA with within subject factors of context (attractive vs unattractive) and ownership (ownership vs no ownership). The analysis revealed a main effect of context $F_{1,59}=18.557, P=0.000$, showing that model presented in attractive contexts was perceived as more attractive than when the same model was presented in the unattractive contexts. There was a main effect of ownership $F_{1,59}=22.937, P=0.000$, implied ownership suppressed attractiveness ratings. However, the context by ownership interaction (predicted by sexual selection theories) was not statistically significant $F_{1,59}=0.490$, $P=0.487$. 


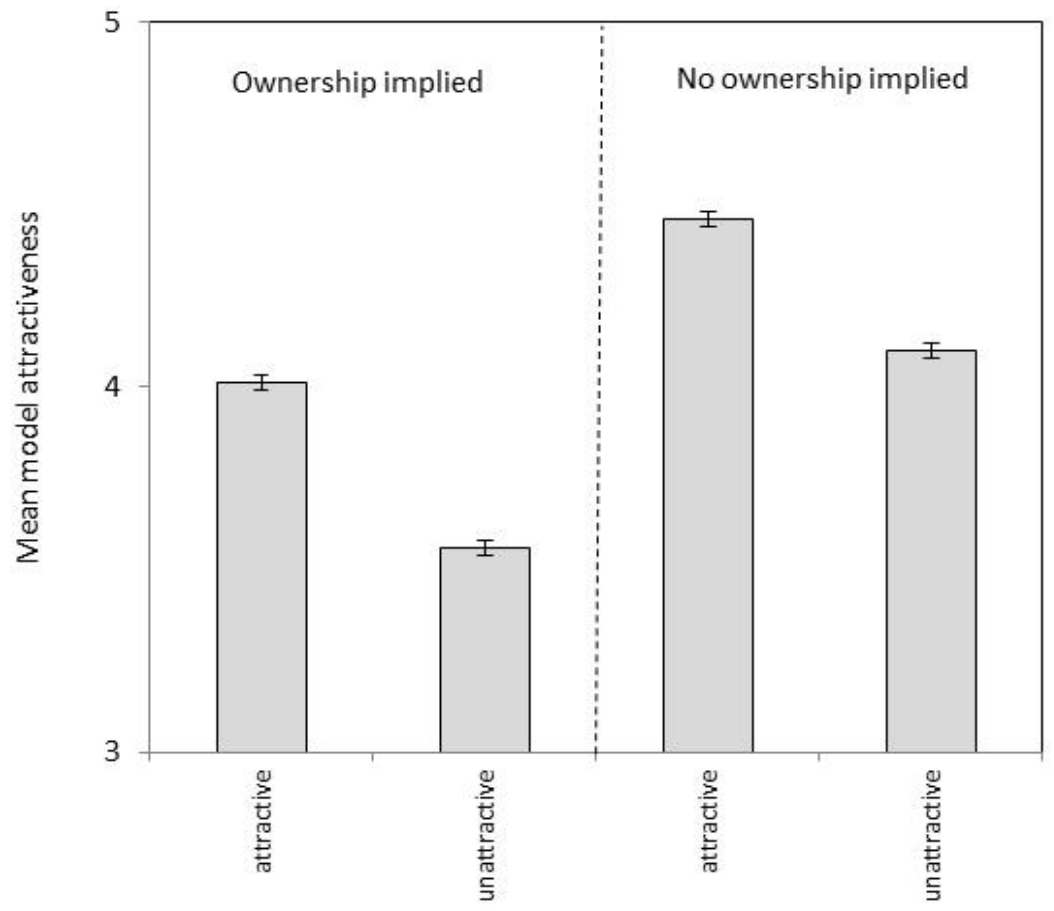

Figure 2. Female adults' mean attractiveness ratings of a male model in attractive and unattractive contexts that either implied ownership or did not. Error bars show the standard error of the mean

\section{INTERIM DISCUSSION}

The findings of Experiment 1 are inconsistent with the notion that heterosexual women's attractiveness ratings of male targets were specifically influenced by implicit material status (e.g., Dunn \& Searle, 2010; Dunn \& Hill, 2014). Instead, women's ratings of the male model increased in both types of attractive context (with and without implied ownership). This finding indicates that for heterosexual women the attractiveness of a male model can change depending upon the attractiveness of the context in which he is encountered. This finding is unsurprising when we take into consideration the wealth of evidence from the cognitive psychology literature showing that attentional resources in men and women are directed differently with respect to central (target) and peripheral (distractor) stimuli. It should be noted that sexually differentiated sensitivity to, and preferences for, specific forms of context and objects develops early in human childhood (under 6 years) and includes (inter alia) sex differences in preference for décor and sex-specific toys (Rheingold \& Cook, 1975). Various ex- 
planations have been offered to account for why boys and girls develop preferences for pink vs blue colour schemes or guns vs dolls as toys, some appeal to a social interactionist perspective (e.g., Cahill, 1983) whilst others stress the general importance of inherited factors in cognition and preferences during infancy (e.g., Baillargeon, 1987). Our analysis does not inform about the origins of how women identify some contexts as attractive (e.g., a large expensive house) and others (e.g., a rubbish tip) as unattractive. All that the current study does is to exploit the fact that adult women will form such judgements and will have responded to our contexts accordingly.

Thus far, our analysis has only brought us to a point where we can conclude that women attend to both background and target and that some aspect of the properties (or value) of the background transfers to the male model target. Earlier we offered evidence that women's attention is automatically captured by background cues. It is appealing to account for women's ratings in Experiment 1 on the basis of peripheral attention because such an account is very simple. One prediction that follows from this simple attentional capture account is that men may be impaired (relative to women) in their ability to attend to and utilise contextual information whenever there is a requirement for them to do so. They would have to overcome their natural tendency to focus on target stimuli and attend instead to both the context and the target on every trial. An alternative and more interesting explanation for why women were influenced by the attractiveness of the background and men were not (e.g., Dunn \& Searle, 2010) could be that men and women may differ in the way that they represent or encode the relationships between contextual and target stimuli, the following discussion is designed to elaborate this point.

For some decades learning theorists have been trying to account for how animals are able to learn discriminations that cannot be solved using simple associative processes. One such discrimination is the so-called biconditional discrimination, the contingencies of which are presented in Tables 1 and 2. It is clear that learning in a biconditional discrimination cannot proceed if the learner only attends to either the target stimulus (X or Y) or the context stimulus (A or $\mathrm{B})$, they must attend to both in order to solve the discrimination because only combinations of cues can act as effective predictors. There is ample evidence that animals (rats and pigeons) can learn such discriminations (e.g., Honey \& Watt, 1998; Honey \& Watt, 1999) but there are still competing explanations regarding how they achieve this (see Bonardi, Bartle, \& Jennings, 2012; George \& Pearce 2012).

Two accounts are now favoured regarding how animals solve biconditional discriminations. The first of these accounts supposes that animals form hierarchical (or occasion setting) representations where target cues form weak associations with outcomes, these associations then require enhancement by addi- 
tional input from representations of context stimuli (Bonardi et al., 2012; see Table 1 lower panel). In a hierarchical model, the target and context cues are represented separately and are functionally distinct, the context is represented in a higher order fashion because it acts on, and subsequently modulates, the value of the target stimuli with which it co-occurs. Occasion setting has been demonstrated when stimuli used as contexts in conditional discriminations are better able (than stimuli not used as contexts) to transfer this modulating property to a new target stimulus (a transfer of occasion setting test, see Holland (1989)).

The second kind of account supposes the operation of an episodic memory process that integrates all stimuli present on any learning trial and encodes them using a configural form of representation (George \& Pearce, 2012; see Table 1 upper panel). According to configural accounts, stimuli themselves do not form direct associations with outcomes, instead configural representations (which integrate all stimuli present on any learning trial) act as episodic representations that can acquire associative strength. Contextual cues do not therefore receive any privileged (hierarchical) processing and are treated in the same way as all other stimuli present on any learning trial.

Table 1. Two different associative structures that can account for the attractiveness ratings provided by the women in Experiment 1 and biconditional discrimination learning in Experiment 2

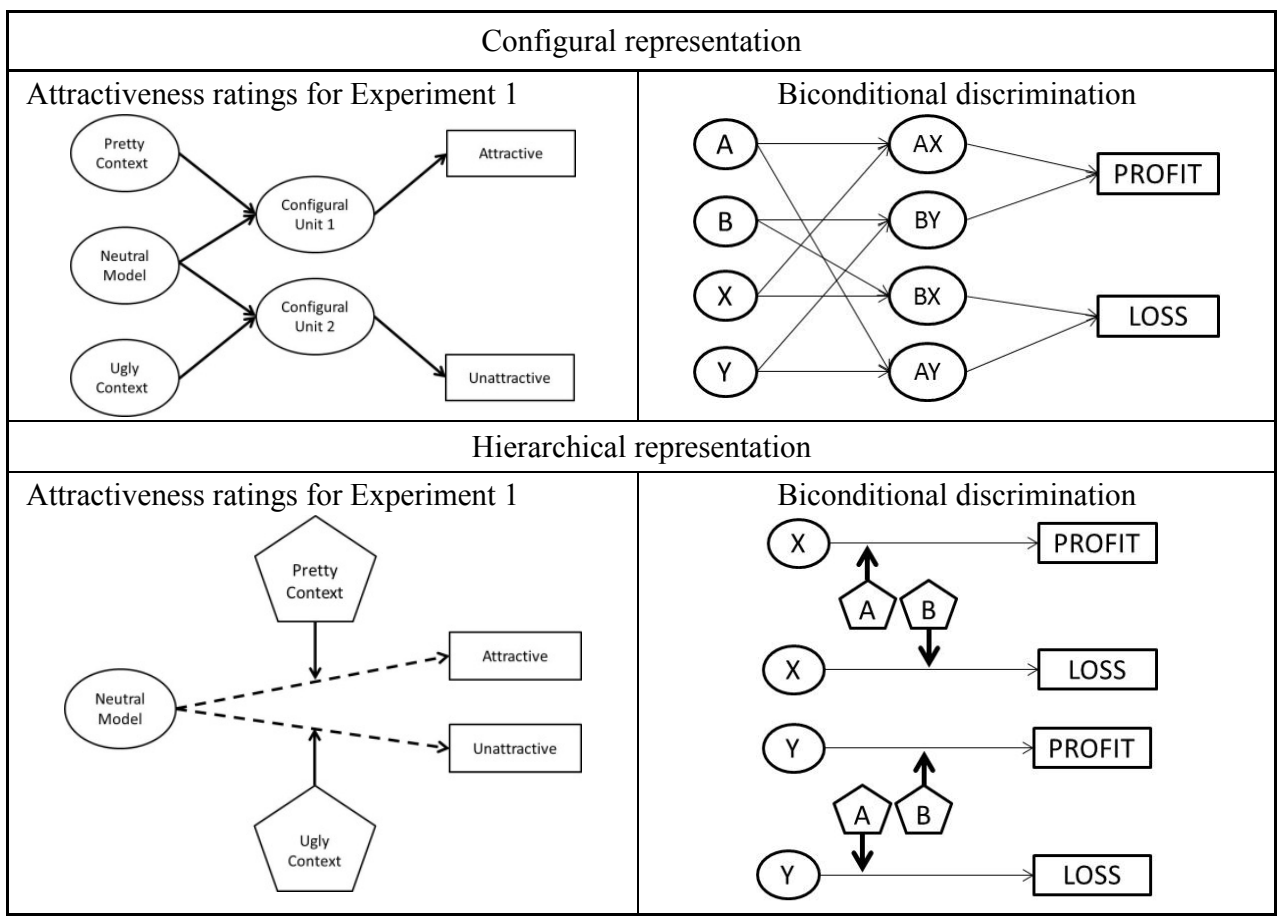




\section{EXPERIMENT 2}

Above we entertained the possibility that men might be relatively impaired in their ability to attend to contextual information. Such a possibility would be supported if men showed poorer learning during biconditional discrimination training because the contingencies of this discrimination require attention to both target and context. Secondly, we considered the possibility that biconditional discriminations can be solved by the engagement of one of two possible forms of representation (hierarchical vs configural). The fact that women (in Experiment 1) rated the attractiveness of male models as higher in attractive contexts is evidence that they must have attended both to the background and the target stimuli. The higher attractiveness ratings in the attractive contexts may have resulted from the operation of either hierarchical or configural representations that encoded the relationship between the target male and properties of the context in which he was presented. If a configural representation had been employed, then increased attractiveness would have resulted from the simple combination (or summation) of attractive features from the context and from the model. Watt and Honey (1997) provide evidence that combining cues that have independently signalled different positive outcomes (sucrose solution and food pellets for a hungry rat) produces more vigorous appetitive behaviour than combinations of cues that have signalled the same positive outcome (either sucrose or food pellets). There is therefore good evidence that summation can be anticipated when cues are configured, if we suppose that the male model and the context (e.g., the beach) are both attractive but differ qualitatively. In contrast to the above configural account, if the women encoded the relationship between the model and the contexts using a hierarchical form of representation (as is implied in sexual selection theory), then the value of the target male would have been conditional (to some degree) upon the attractiveness of the context. One way to differentiate between these competing accounts would be to employ a transfer of occasion setting test (as outlined above). If either group shows occasion setting transfer, then we can conclude that they tend to encode biconditional relationships in a hierarchical fashion. If either group 'fails' the transfer of occasion setting test, then we must deduce that they employ configural representations.

The first purpose of Experiment 2 was to determine if men have a general context processing decrement. The first discrimination (stage 1) was used as a measure of men and women's sensitivity to contextual information, allowing comparison of the rates at which these 2 groups learned the discrimination. 
Table 2. Design of Experiment 2, context stimuli were presented on the left of the screen, target stimuli were presented on the right. Participants received feedback (profit or loss) after they had provided a confidence rating on every learning and test trial

\begin{tabular}{|c|c|c|c|c|c|}
\hline \multicolumn{3}{|c|}{\begin{tabular}{c} 
Stage 1 \\
\multicolumn{2}{|c|}{ Biconditional discrimination }
\end{tabular}} & \multicolumn{3}{c|}{$\begin{array}{c}\text { Stage 2 } \\
\text { Occasion setting test }\end{array}$} \\
\hline Context & Target & Outcome & Context & Target & Outcome \\
\hline \multirow{2}{*}{ A } & X & Profit & B & P & Profit \\
& Y & Loss & Q & Loss \\
\hline \multirow{2}{*}{ B } & X & Loss & X & P & Loss \\
& Y & Profit & & Q & Profit \\
\hline
\end{tabular}

The second purpose of Experiment 2 was to determine whether men and women tend to encode contextual information in qualitatively different ways by applying a test for transfer of occasion setting. Stage 2 involved a second biconditional discrimination that used 2 cues $(\mathrm{B} \& \mathrm{X})$ from stage 1 as contexts, one had previously served as a context (B) whilst the other had served as a target (X). Stage 2 also introduced 2 novel target stimuli (P \& Q). If participants processed the relationships between $\mathrm{A}, \mathrm{B}, \mathrm{X} \& \mathrm{Y}$ hierarchically in stage 1 , then $\mathrm{B}$ should transfer conditional control more readily than $\mathrm{X}$ when used in new contexttarget associations.

\section{METHOD OF EXPERIMENT 2}

\section{Participants}

Ninety seven undergraduate Psychology students acted as participants (65 were female and 32 male), mean age for the sample was 22.5 and ranged between 19 and 56 years. All students were native English speakers. Participants were fully debriefed about the design and purpose of the study after completion of the experiment.

\section{Procedure}

A Visual Basic program was used to give instructions, present stimuli, provide feedback and record responses. Male and female participants completed the experiment together in a teaching laboratory containing 32 PCs. On entering the laboratory, participants were directed to sit at a computer. Alternate computers were used, so that none of the participants were sitting directly next to another, 
this was to minimise distraction between participants. The participants were tested in 'batches' which ranged in size from 7 to 15 at a time.

Table 2 shows the combinations of commodities and the trial outcomes employed during the 2 stages of the experiment. Contextual stimuli were always presented on the left of the screen whilst target cues were presented on the right. Pairs of context and target stimuli were always presented simultaneously. Participants were not instructed about the spatial location of cues but this contextleft and target-right arrangement was chosen to exploit the natural tendency of English speakers to read from left to right, therefore perceiving and processing the cues on the left first, so that these might act as contextual cues that would 'set the occasion' for processing of target cues to the right.

In Table 2, stimuli A, B, X, Y P, \& Q represent names for fictitious trading commodities (e.g., rice or steel). They were randomly assigned from a pool of possible commodities. During stage 1 combinations AX and BY were paired with profit whilst AY and BX were paired with loss. Stage 1 therefore represents the standard contingencies of a biconditional discrimination.

Participants received a fixed number of 80 training trials during stage 1. The 4 types of trial (Table 2) were each presented once in 20 consecutive cycles of training trials. The order of trials within each cycle was randomised. On each trial the participants were presented with a context-target pair of cues and were required to provide a rating (by selecting a number on the computer keyboard) that reflected their confidence that one of two possible outcomes would occur at the end of the trial. The rating scale ranged between 1 (absolute confidence of LOSS) through 5 (uncertainty) to 9 (absolute confidence of PROFIT). Each trial was terminated with feedback to the participants indicating the outcome on that trial, the outcome of each trial was independent of the participants' response. Participants' responses always initiated the next trial immediately. Stage 2 of the experiment commenced immediately and automatically at the end of stage 1, participants were not warned or briefed about the new stimuli or task requirements.

In Stage 2 participants each received 40 trials of training on a new biconditional discrimination that used $\mathrm{P} \& \mathrm{Q}$ as targets and $\mathrm{B} \& \mathrm{X}$ as context stimuli (see Table 2). The 40 trials of stage 2 were arranged in 10 consecutive blocks of 4 trials. In each block the order of the 4 different types of trial (see Table 2) was randomised. In accordance with the contingencies of a biconditional discrimination, half of the trials were paired with the profit outcome (BP \& XQ), the remaining trials (BQ \& XP) ended with the loss outcome. 


\section{RESULTS OF EXPERIMENT 2}

\section{Stage 1 discrimination training}

Figure 3 presents the biconditional discrimination learning data for male and female participants in the first stage of Experiment 2. Learning of the biconditional discrimination was indexed as the mean difference in confidence ratings between profit trials (AX \& BY) and loss trials (AY \& BX). Inspection of Figure 3 indicates that the males showed moderately higher discrimination scores. This impression of the acquisition data was not confirmed by a $2 \times 5,2$ -

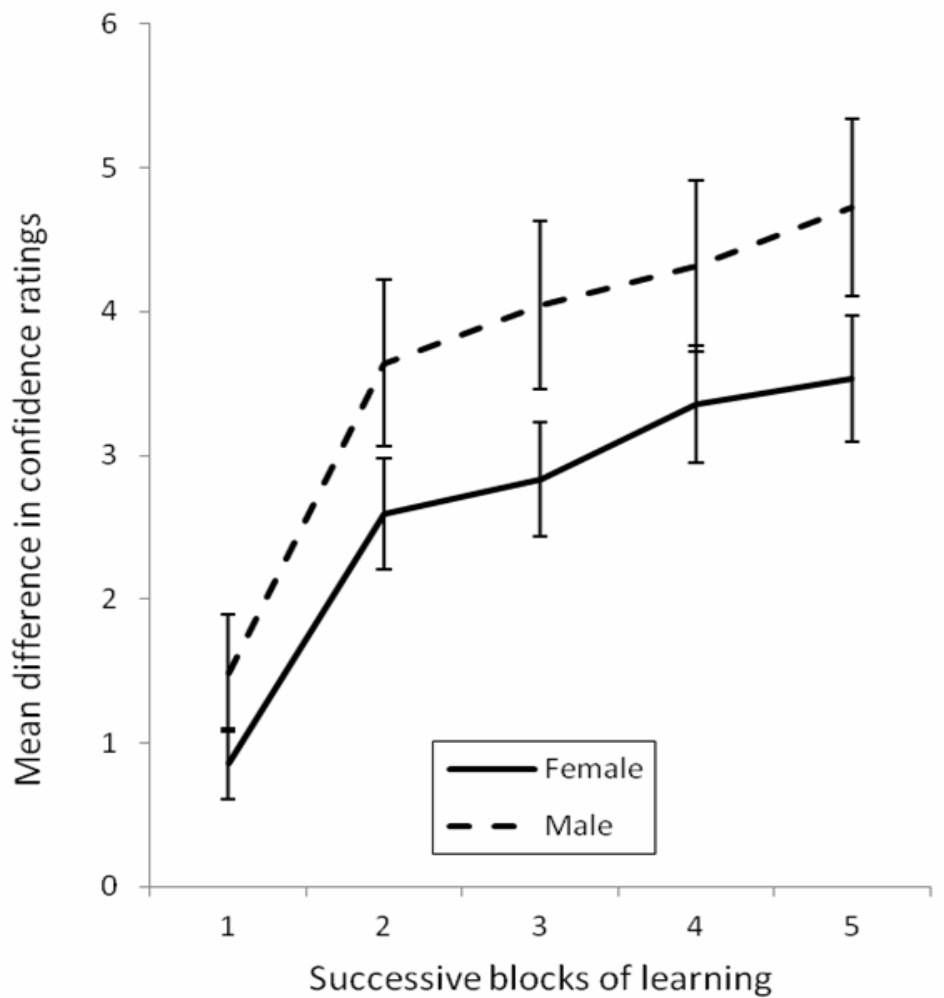

Figure 3. Mean difference in confidence ratings between profit (AX \& BY) and loss trials (AY \& BX) across the biconditional discrimination training blocks of stage 1 in Experiment 2. Error bars present the standard error of the mean

way ANOVA that compared mean differences in confidence ratings between men and women across the 5 successive blocks of training trials in stage 1 . There was a main effect of time $F_{4,380}=45.05, P=0.000$, no effect of sex 
$F_{1,95}=2.87, P>.05$ and no interaction between these factors $F_{4,380}=0.46$, $P=0.756$. The males and females therefore acquired the discrimination at the same rate and performed equivalently throughout. It is important to note that similarity in the performance of the 2 groups simplified interpretation of the results from the second stage of the experiment. Specifically, any sex differences observed in stage 2 could not be explained by carry-over of sex differences from acquisition of the baseline discrimination in stage 1 .

\section{Transfer of occasion setting test}

The following series of analyses were designed to test the possibility that men and women may differ in the way that they represent contextual information. The procedure was analogous to tests used in the animal learning literature that have explored the nature of associations formed during so-called non-linear discrimination learning (Bonardi, et al., 2012; Honey \& Watt, 1998, 1999), such as the biconditional discrimination in Stage 1 of this experiment.

Acquisition of a new discrimination will have worked against observation of the transfer of occasion setting effect because new and competing associations were being formed. The following analyses therefore focused on the first 4 trial cycles of the transfer test, transfer effects were not anticipated in later trials, so the data for the remaining trials in the transfer test were discarded. Please note that each cycle of training trials contained one each of the four test trial types (BP, BQ, XP, \& XQ), the following analyses therefore reflect detailed examination of trial-by-trial learning.

Figure 4 presents the results from the transfer test. It is clear from the right panel that the males did not show any transfer of occasion setting, whilst the predicted disadvantage for $\mathrm{X}$ relative to $\mathrm{B}$ is apparent in the test performance of the female group in the third and fourth cycles of trials.

The next analysis was a 3-way ANOVA that included sex (male or female) as a between subjects factor, time $\left(1^{\text {st }}, 2^{\text {nd }}, 3^{\text {rd }}\right.$ and $4^{\text {th }}$ cycles of test trials $)$ as a within subjects factor and stimulus ( $\mathrm{B}$ or $\mathrm{X})$ as a second within subjects factor. There was a main effect of time $F_{3,285}=18.08, P<0.001$ that interacted with the sex factor $F_{3,285}=3.94, P<.001$. This result indicates that overall there was an increase in discriminative control across the first 4 cycles of the test and that the groups differed in the magnitude of this increase. Post hoc analyses that used Bonferroni adjustments for multiple comparisons provided only tentative support for this interpretation, the overall discrimination performance differed 


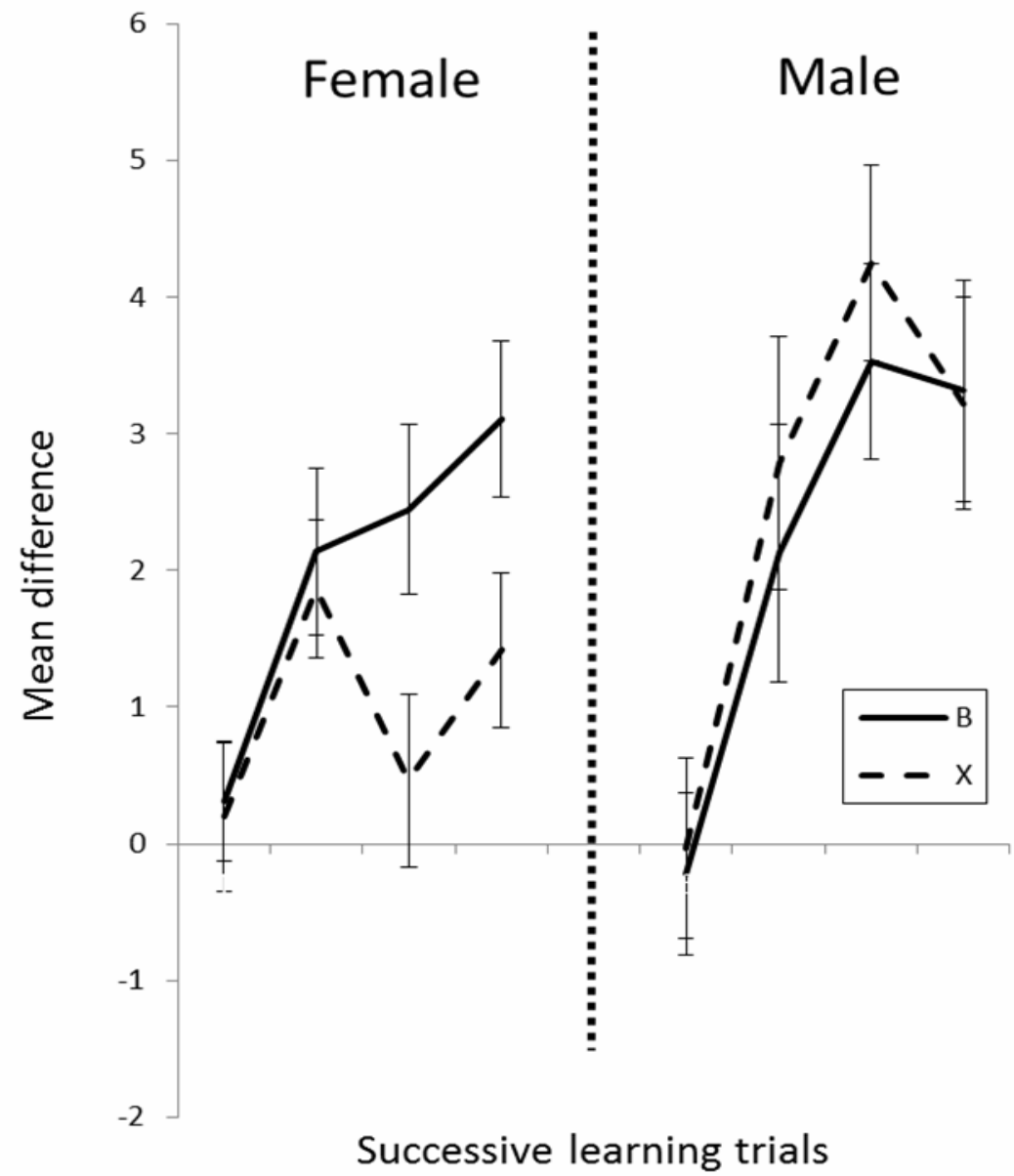

Figure 4. Mean discriminative control for stimuli B and X are presented for the $1^{\text {st }}, 2^{\text {nd }}, 3^{\text {rd }} \& 4^{\text {th }}$ cycles of occasion setting transfer test trials. Stimulus control for B was calculated as the mean difference in confidence ratings between profit trials (BP) and loss trials (BQ). Stimulus control for $\mathrm{X}$ was calculated as the mean difference in confidence ratings between profit trials $(\mathrm{XQ})$ and loss trials (XP). Error bars present the standard error of the mean

between the males and females only on the $3^{\text {rd }}$ cycle of trials. The critical comparison for this analysis was the 2-way interaction between the sex and stimulus factors, such an interaction would indicate that one of the groups had shown transfer of occasion setting (a difference in learning with B vs X) whilst the other had not. There was in fact a 2-way interaction between these factors $F_{1,95}=4.455, P<.05$. Pairwise comparisons with Bonferroni adjustment for 
multiple comparisons indicated that discriminative control by $\mathrm{X}$ was inferior to that for $\mathrm{B}$ in the female group (mean difference $=1.015, P<.01$ ), whilst $\mathrm{B}$ and $\mathrm{X}$ controlled similar levels of discriminative control in the test performance of the males (mean difference $=-0.367, P>.05$ ). All other main effects and interactions in the above 3-way ANOVA failed to satisfy the alpha criterion, all $F_{S}<1$.

\section{DISCUSSION OF EXPERIMENT 2}

Experiment 2 employed a learning task to examine 1) whether men and women differed in their sensitivity to contextual information and 2) whether men and women differed in the associative structures that they employ to encode the relationships between contexts and target stimuli.

We found no evidence for a context processing decrement in men, they were equally able (as the women) to utilise contextual information when the task required them to (in a biconditional discrimination). However, the evidence from stage 2 of Experiment 2 indicated that men tended to encode the relationships between contextual and target stimuli configurally (George \& Pearce, 2012), whilst women encoded the same relationships hierarchically (Bonardi, et al., 2012). It seems therefore that the fundamental difference between men and women is not in contextual attention per se (Merritt et al., 2007; Yarney, 2004), nor is it necessarily in attention to cues indicating elevated material status (Dunn \& Searle, 2010; Dunn \& Hill, 2014), but is instead better characterised by a difference in the sexes' tendency to infer or impose hierarchical associations between contexts and the target stimuli with which they are paired. The women in our sample appeared to use context information to disambiguate the value of the target stimuli, whilst men attended to all relevant stimuli on each learning trial, processed all cues equivalently and integrated them into configural episodic representations. It is important to note that the stimuli were selected randomly to act as targets and contexts, the initial relations between words used as stimuli were therefore completely arbitrary for both the men and the women.

\section{GENERAL DISCUSSION}

Experiment 1 included conditions to control for the specific influence of material status indicators on women's attractiveness ratings for a male model, this was achieved by including and excluding them from contexts that were either attractive or unattractive. This design allowed examination of the specificity of sexual selection accounts which suppose that women have evolved with traits 
that encourage them to modulate attractiveness ratings on the basis of men's apparent material status (Buss, 1989). No evidence was found to support specific status cue sensitivity because women's ratings increased in both attractive contexts (with and without implied high status ownership). This pattern of data is consistent with female performance reported elsewhere in tasks measuring vigilance (Merritt et al., 2007), attention (Giambra \& Quilter, 1989) and episodic memory (Yarney, 2004).

All of the effects thus far (including those employing material status cues) can be readily reconciled in terms of sex differences in automatic attention toward, and utilisation of, contextual stimuli. This account has considerable appeal because a wide range of effects, collected in order to explore quite different phenomena, can be accommodated in a single explanation. However, this account leaves some residual questions unanswered. Firstly, are men simply blind to context or are they more able than women to selectively ignore the background? If men are less able to process background cues, then we would anticipate that they would have difficulty doing this in any task that requires attention to both background and target stimuli. Secondly, sexual selection accounts of how women perform attractiveness assessments must suppose the operation of 2 distinct sub-processes. The first is that women are sensitive to valuable features of the background and the second is that they use this information to modify their assessment of targets placed in the background. The combined effect of these 2 processes is to make the value of a male target (to some extent) conditional upon the meaning (or value) of the background. What might be supposed therefore is that men and women may differ in the nature of the associative structures that they employ to encode the relationships between background and target stimuli. Specifically, men and women may differ in their tendency to infer conditional associations between backgrounds and targets.

Experiment 2 was designed to resolve these residual questions. We devised a novel fictitious stockbroker learning task which was based on animal learning tests for associative structures formed during conditional discrimination learning (i.e. transfer of occasion setting; Bonardi, Bartle, \& Jennings, 2012; Holland, 1989; see Table 1). Men were not simply blind to context and were able to learn the biconditional discrimination (which required contextual and target attention) as rapidly as the women. In stage 2 women showed a bias in their learning of a second biconditional discrimination; their learning favoured a context stimulus that had previously been used as a context, they showed transfer of occasion setting. Men were unaffected by the roles (context or target) that the stimuli had served in the first stage of the experiment.

Transfer of occasion setting is widely interpreted as evidence for the involvement of a hierarchical form of representation (Holland, 1989) and we conclude that women tend to encode the relationships between background and tar- 
get stimuli in this way, as sexual selection theories will generally suppose that they must (in mate selection choices). Men showed no evidence for hierarchical representations and we conclude (by deduction) that the only other viable form of representation that can account for their learning in a biconditional discrimination is a process of configuration (George \& Pearce, 2012; Pearce, 1987).

We must acknowledge that there were some inherent weaknesses in the materials that we employed in Experiment 1. Previous studies manipulating implied ownership have placed models inside desirable (high status) cars (Dunn \& Searle, 2010) or apartments (Dunn \& Hill, 2011) and as such it could be argued that they have made implied ownership more explicit than the materials in Experiment 1 here. It could therefore be argued that our materials are more like holiday snaps and the effects that we have observed might not generalise to situations in which ownership is more explicitly implied.

The purpose of the current paper was not to challenge the general validity of sexual selection. Instead we sought to explore whether fundamental sex differences in contextual representation might reveal a basic cognitive process that could potentially account for sex differences reported in the heterosexual human mate selection literature (e.g., Dunn \& Searle, 2010; Dunn \& Hill, 2014). We do not feel that we are in a position to offer explanations based on the evolutionary pressures (in our ancestral past) that have resulted in men and women differing in terms of the way that they attend to, utilise and represent the relationships between events in their world, we will leave others to do this. What we hope we have offered instead is an introduction to an alternative way to describe and understand some basic cognitive differences between men and women and how these differences may provide alternative accounts of some sexual selection phenomena. Even at this descriptive level the differences that we have uncovered must have a pervasive impact on the way that adult male and female humans perceive and interact with each other, both in the context of sexual attraction and outside of it. Indeed, our findings indicate that men and women may differ ubiquitously in the way that they represent relationships between all of the stimuli and contexts that they encounter.

\section{REFERENCES}

Aharon, I., Etcoff, N., Ariely, D., Chabris, C. F., O'Connor, E., \& Breiter, H. C. (2001). Beautiful faces have variable reward value: fMRI and behavioural evidence. Neuron, 32, 537-551.

Baillargeon, R. (1987). Young infants' reasoning about the physical and spatial properties of hidden object. Cognitive Development, 2, 179-200.

Bayliss, A. Pellegrino,G., \& Tipper, S. (2005). Sex differences in eye gaze and symbolic cueing of attention. The Quarterly Journal of Experimental Psychology, 58, 1-17.

Bonardi, C., Bartle, C., \& Jennings, D. (2012). US specificity of occasion setting: Hierarchical or configural learning. Behavioural Processes, 90, 311-322. 
Buss, D. M. (1989). Sex differences in human mate preferences: Evolutionary hypotheses testing in 37 cultures. Behavioral and Brain Sciences, 12, 1-49.

Buss, D., \& Schmitt, D. (1993). Sexual strategies theory: An evolutionary perspective on human mating. Psychological Review, 100, 204-232.

Cahill, S. E. (1983). Reexamining the acquisition of sex roles: A social interactionist approach. Sex Roles, 9, 1-15.

Collins D. W., \& Kimura, D. (1997). A large sex difference on a two-dimensional mental rotation task. Behavioural Neuroscience, 111, 845-849.

Dunn, M. J., \& Hill, A. (2014). Manipulated luxury-apartment ownership enhances opposite-sex attraction in females but not males. Journal of Evolutionary Psychology, 12, 1-17.

Dunn, M., \& Searle, R. (2010). Effect of manipulated prestige-car ownership. British Journal of Psychology, 101, 69-80.

Furnham, A., Swami, V., \& Shah, K. (2006). Body weight, waist-to-hip ratio and breast size correlates of ratings of attractiveness and health. Personality and Individual Differences, 41, 443-454.

George, D. N., \& Pearce, J. M. (2012). A configural theory of attention and associative learning. Learning and Behavior, 40, 241-254.

Giambra, L. M., \& Quilter, R. (1988). Sustained attention in adulthood: A unique, large-sample, longitudinal and multicohort analysis using the Mackworth Clock-Test. Psychology and Aging, 3, 75-83.

Gibbins, R. (1969). Communications aspects of women's clothes and their relationship to fashionability. British Journal of Social and Clinical Psychology, 8, 301-312.

Halpern, D. F. (2000). Sex differences in cognitive abilities (3rd Edition). Lawrence Erlbaum, Associates, Inc. Publishers, Mahwah, NJ.

Hassebrauk, M. (1998). The visual process method: A new method to study physical attractiveness. Evolution and Human Behavior, 19, 111-123.

Hill, E., Nocks, E., \& Gardner, L. (1987). Manipulation by physical and status displays. Ethology and Sociobiology, 8, 43-154.

Holland, P. C. (1989). Acquisition and transfer of conditional discriminative performance. Journal of Experimental Psychology: Animal Behavior Processes, 15, 154-165.

Honey, R. C., \& Watt, A. (1998). Acquired relational equivalence: Implications for the nature of associative structures. Journal of Experimental Psychology: Animal Behavior Processes, $24,325-334$.

Honey, R. C., \& Watt, A. (1999). Acquired relational equivalence between contexts and features. Journal of Experimental Psychology: Animal Behavior Processes, 25, 324-333.

Hoult, T. F. (1954). Experimental measurement of clothing as a factor in some social ratings of selected American men. American Sociological review, 19, 326-327.

Marritt, P, Hirshman, E., Wharton, W., Stangl, B., Devlin, J., \& Lenz, A. (2007). Evidence for gender differences in visual selective attention. Personality and Individual Differences, 43, 597-609.

Pearce, J. M. (1987). A model of stimulus generalization for Pavlovian conditioning. Psychological Review, 94, 61-73.

Rheingold, H. L., \& Cook, K. V. (1975). The content of boys' and girls' rooms as an index of parents' behaviour. Child Development, 46, 459-463.

Townsend, J. M. (1989). Mate selection Criteria: A pilot study. Ethology and Sociobiology, 10, 241-253.

Tractinsky, N. Cokhavi, A. Kirschenbaum, M. (2004). Using ratings and response latencies to evaluate the consistency of immediate aesthetic perceptions of web pages. Proceedings of the Third Annual Workshop on HCI Research, Washington, D.C., December 10-11. 
Trivers, R. L. (1972) Parental investment and sexual selection. In B. Campbell, (Ed) Sexual selection and the descent of man, 1871-1971, Aldine-Atherton, Chicago, pp. 136-179.

Watt, A., \& Honey, R. C. (1997). Combining CSs associated with the same or different USs. The Quarterly Journal of Experimental Psychology, 50B, 350-367.

Yarmey, A. D. (1986). Verbal, visual, and voice identification of a rape suspect under different levels of illumination. Journal of Applied Psychology, 71, 363-370.

Yarmey, A. D. (2004). Eyewitness recall and photo identification: A field experiment. Psychology, Crime \& Law, 10, 53-68.

Yonker, J. E., Eriksson, E., Nilsson, L.-G., \& Herlitz, A. (2003) Sex differences in episodic memory: the minimal influence of estradiol. Brain and Cognition, 52, 231-238. 